\title{
Disturbance Attenuation and Rejection for a Class of Switched Nonlinear Systems Subject to Input and Sensor Saturations
}

\author{
Yunliang Wei $\mathbb{D},{ }^{1,2}$ Liping Sun, ${ }^{1}$ Shengsen Jia, ${ }^{1}$ Kunming Liu, ${ }^{1}$ and Fanwei Meng ${ }^{1}{ }^{1}$ \\ ${ }^{1}$ School of Mathematical Sciences, Qufu Normal University, Qufu, Shandong 273165, China \\ ${ }^{2}$ School of Engineering, University of South Wales, Cardiff, CF37 1DL, UK \\ Correspondence should be addressed to Yunliang Wei; xllweiyunliang@gmail.com
}

Received 28 November 2018; Revised 13 March 2019; Accepted 19 March 2019; Published 16 April 2019

Academic Editor: Alessandro Lo Schiavo

Copyright (c) 2019 Yunliang Wei et al. This is an open access article distributed under the Creative Commons Attribution License, which permits unrestricted use, distribution, and reproduction in any medium, provided the original work is properly cited.

\begin{abstract}
This paper investigates the problem of disturbance attenuation and rejection for a class of switched nonlinear systems subject to input and sensor saturations, in which exosystem generated disturbances and $\mathrm{H}_{2}$-norm bounded disturbances are considered. The full-order and reduced-order observers are designed according to whether the system states are available or not. Based on the estimating values of the system states and exosystem generated disturbances, the design schemes for the composite controllers are put forward based on the full-order and reduced-order observers, respectively. For a switched system, the input and sensor saturations would influence the effective synthesis of observer and controller. By sector nonlinearity technology, the stability of the augmented closed-loop systems under the proposed composite controllers are analyzed, and the conditions of synthesis of the observers and controllers are further presented to ensure the augmented systems to be robustly asymptotically stable with a weighted $H_{\infty}$ performance level. An example is given to guarantee the effectiveness of the proposed control schemes.
\end{abstract}

\section{Introduction}

In past decades, the research on switched systems has attracted more and more attentions [1,2]. Switched systems often are encountered in practical control fields, such as mechanical control, aircraft and air traffic control, and switching power converter, which consist of a finite number of subsystems and a switching law that orchestrates the switching between these subsystems [3]. The most important research direction of switched systems is to investigate the stability of switched systems. There are two aspects of this problem: one is how to obtain stable condition of switched systems under the arbitrary switching law [4-6]; the other is how to construct a switching law to guarantee the switched systems to be stable $[7,8]$. Based on the discussion about the stability, some schemes of controller synthesis have been further put forward $[9,10]$. Recently, many excellent results on the stability analysis and synthesis of discrete-time switched systems have been established [11, 12].

The disturbances are mainly caused by measurement noise, load changing, external surroundings, sensor and actuator errors, modeling errors, and structural changes in many actual processes. In recent years, the attenuation and rejection of disturbances have always been hot research topics. Various ways have been tried to deal with disturbances, so that the performance of the system could be less degraded and the system could be more stable [13-23]. In [13], a robust adaptive full-order observer is proposed with adaptation laws for the unknown disturbances and parameters, and a reduced-order observer-based response system is developed under the same assumptions, which can synchronize part of states of drive system. In [14], the problem of composite antidisturbance control for time-varying delay Markovian jump nonlinear systems with multiple disturbances is investigated; by combining disturbance observer and $L_{2}-L_{\infty}$ control method, the disturbances are attenuated and rejected. Recently, a composite fault-tolerant control with disturbance observer scheme is considered for a class of stochastic systems with faults and multiple disturbances. A stochastic disturbance observer is designed to estimate exogenous disturbances and a composite fault-tolerant controller is proposed to compensate disturbances and faults [15]. A hierarchical antidisturbance control methodology is given for Markovian jump systems with nonlinearity and multiple disturbances [16]. It is noteworthy 
that how to restrain disturbance more effectively for switched systems is still an open and attractive research direction.

It is well known that actuator saturation, a quite common and inherent phenomenon for practice controlled systems, will lead to poor performance of the closed-loop system and may even cause the system to lose its stability. Therefore, in the past few decades, the study of saturated system has attracted considerable attentions [24-32]. In [25], an antiwindup design is proposed to compensate the stabilizing controller for a linear system subject to nested input saturation, such that the negative effects from saturation are eliminated. In [26], the robust finite-horizon output feedback control problem for a class of uncertain discrete stochastic nonlinear time-varying systems with both sensor and actuator saturations is investigated. An output feedback controller is proposed such that the $H_{\infty}$ disturbance attenuation level is guaranteed for the nonlinear stochastic polytopic system in the presence of saturated sensor and actuator outputs. Recently, the stabilization problem of a class of nonlinear systems with actuator saturation is investigated via active disturbance rejection control (ADRC) and a LMIbased algorithm is correspondingly established to design the linear ADRC controller [27]. Further, in [28], a new compensation scheme is proposed to attenuate the effects from both the randomly occurring faults and the sensor saturations onto the estimation performance. Recently, the optimal control, antiwindup control, and model predictive control have become the most basic methods to deal with saturation problems $[33,34]$.

Motivated by the above-mentioned literatures, this paper investigates the problem about analysis and synthesis of a class of switched nonlinear systems with input and sensor saturations. In the past few decades, the researchers only considered switching and input saturation [29, 30, 35] or only considered input and sensor saturation [26]. When a switched system is subject to both input and sensor saturation, the analysis and synthesis would be very difficult. The main contribution and innovation in this paper are included as follows: firstly, the full-order and reduced-order observers are designed to the values of the system states and matched disturbances, respectively; secondly, by sector nonlinearities technology, the design conditions of the controller ensuring the augmented system is robustly asymptotically stable in the absence of the mismatched disturbances for all initial conditions under arbitrarily switching law; thirdly, a $H_{\infty}$ control scheme is presented to attenuate the mismatched disturbances for the augmented closed-loop system.

\section{Problem Formulation}

Consider the following discrete-time switched system in presence of nonlinearities and saturation constraints

$$
\begin{aligned}
x(k+1)= & A_{\sigma(k)} x(k)+B_{\sigma(k)} \text { sat }\left[u(k)+d_{1}(k)\right] \\
& +G_{\sigma(k), 1} f_{\sigma(k), 1}(x(k), k)+E_{\sigma(k), 1} d_{2}(k) \\
y(k)= & \operatorname{sat}\left(C_{\sigma(k)} x(k)\right)+G_{\sigma(k), 2} f_{\sigma(k), 2}(x(k), k) \\
& +D_{\sigma(k)} d_{1}(k)+E_{\sigma(k), 2} d_{2}(k),
\end{aligned}
$$

where $x(k) \in R^{n}, u(k) \in R^{m}$, and $y(k) \in R^{p}$ are the state, input, and output vectors of the system, respectively; $d_{2}(k) \in R^{w}$ is the disturbance which belongs to $\mathscr{L}_{2}[0, \infty)$. $\sigma(k): Z \longrightarrow \mathbf{I}[1, q]$ is the switching law. When $\sigma(k)=i \epsilon$ $\mathbf{I}[1, q]$, the $i_{\text {th }}$ subsystem is activated. For the sake of brevity, in the following, we assume that $\sigma(k)=i$. The matrices $A_{i}$, $B_{i}, C_{i}, D_{i}, G_{i 1}, G_{i 2}, E_{i 1}$, and $E_{i 2}$ are known, real matrices of appropriate dimensions. $d_{1}(k) \in R^{m}$ is the unknown disturbance satisfying the following assumption.

Assumption 1. The disturbance $d_{1}(k)$ in the control input path can be formulated by the following exogenous system:

$$
\begin{aligned}
\omega(k+1) & =W_{\sigma(k)} \omega(k)+M_{\sigma(k)} d_{3}(k) \\
d_{1}(k) & =V_{\sigma(k)} \omega(k),
\end{aligned}
$$

where $W_{i} \in R^{r \times r}, M_{i} \in R^{r \times l}$, and $V_{i} \in R^{m \times r}$ are known matrices for $\sigma(k)=i \in \mathbf{I}[1, q] . d_{3}(k) \in R^{l}$ is supposed to belong to $\mathscr{L}_{2}[0, \infty)$.

Remark 2. In practical engineering, many kinds of disturbances can be described as the dynamic system (2), such as the constant and harmonics noises with unknown phase and magnitude. The systems with disturbances generated by the exogenous model have been widely researched. In (2), $d_{3}(t)$ is an additional disturbance which results from the perturbations and uncertainties of the exogenous model [16].

For further discussion, we need to make an assumption as follows.

Assumption 3. For any $i \in \mathbf{I}[1, q],\left(A_{i}, B_{i}\right)$ is controllable and $\left(W_{i}, B_{i} V_{i}\right)$ is observable.

Definition 4. A nonlinearity $\Psi: R^{j} \longrightarrow R^{j}$ is said to satisfy a sector condition if $\Psi(0)=0$ and

$$
\begin{aligned}
& {\left[\Psi(x)-\Psi(y)-H_{1}(x-y)\right]^{T}} \\
& \quad \cdot\left[\Psi(x)-\Psi(y)-H_{2}(x-y)\right] \leq 0, \quad \forall x, y \in R^{j},
\end{aligned}
$$

where $H_{1}$ and $H_{2}$ are known constant matrices, and $H=H_{1}-$ $\mathrm{H}_{2}$ is positive definite matrix. It is customary that $\Psi$ is said to belong to the sector $\left[H_{1}, H_{2}\right]$.

Remark 5. The nonlinearities $f_{i 1}(x(k), k)$ and $f_{i 2}(x(k), k)$, without loss of generality, are assumed to belong to the sectors $\left[U_{i 11}, U_{i 12}\right]$ and $\left[H_{i 21}, H_{i 22}\right]$, respectively.

Remark 6. In the most exiting results, the system nonlinearities are assumed to satisfy the Lipschitz condition. The sector nonlinearity can contain the Lipschitz nonlinearity by choosing $H_{1}=H_{2}$ to be positive definite matrix, which means that the sector nonlinearity is quite general.

$\operatorname{sat}(\cdot): R^{i} \longrightarrow R^{i}$ denotes the vector valued standard saturation function, which is described as $\operatorname{sat}(v)=\left[\operatorname{sat}_{1}\left(v_{1}\right)\right.$, $\left.\operatorname{sat}_{2}\left(v_{2}\right), \cdots, \operatorname{sat}_{i}\left(v_{i}\right)\right]^{T}$ with $\operatorname{sat}_{j}\left(v_{j}\right)=\operatorname{sign}\left(v_{j}\right) \max \left\{1,\left|v_{j}\right|\right.$, $j \in \mathbf{I}[1, i]\}$. Without loss of generality, we assume that the saturation level is unity. 
Assuming that there exist the diagonal matrices $R_{i 11}, R_{i 12}$ and $R_{i 21}, R_{i 22}$ such that $0 \leq R_{i 11}<I \leq R_{i 12}$ and $0 \leq R_{i 21}<$ $I \leq R_{i 22}$, then the saturation functions $\operatorname{sat}(\bar{u}(t))$ and $\operatorname{sat}(y(k))$ can be rewritten as

$$
\begin{aligned}
\text { sat }(\bar{u}(k)) & =R_{i 11} \bar{u}(k)+\Psi_{i 1}(\bar{u}(k)) \\
\text { sat }\left(C_{i} x(k)\right) & =R_{i 21} C_{i} x(k)+\Psi_{i 2}\left(C_{i} x(k)\right),
\end{aligned}
$$

where $\bar{u}(k)=u(k)+d_{1}(k), \Psi_{i 1}(\bar{u}(k))$ and $\Psi_{i 2}(y(k))$ are nonlinear functions and belong to $\left[0, R_{i 1}\right]$ and $\left[0, R_{i 2}\right]$, respectively, with $R_{i 1}=R_{i 12}-R_{i 11}$ and $R_{i 2}=R_{i 22}-R_{i 21}$, i.e., $\Psi_{1}\left(u(t)+d_{1}(t)\right)$ and $\Psi_{2}(y(t))$ satisfies the following inequalities:

$$
\begin{array}{r}
\Psi_{i 1}(\bar{u}(k))\left(\Psi_{i 1}(\bar{u}(k))-R_{i 1} \bar{u}(k)\right) \leq 0 \\
\Psi_{i 2}\left(C_{i} x(k)\right)\left(\Psi_{i 2}\left(C_{i} x(k)\right)-R_{i 2} C_{i} x(k)\right) \leq 0,
\end{array}
$$

Define a polyhedral set

$$
\mathscr{L}\left(R_{i 11} \bar{u}(k)\right)=\left\{\left|R_{i 11} \bar{u}(k)\right|_{l} \leq 1, l \in \mathbf{I}[1, m]\right\} .
$$

Remark 7. For saturation nonlinearity, the equivalent form (4) comprising linear term and sector nonlinear term is frequently used and valid. Supposing $K_{i}, i=\mathbf{I}[1, q]$ to be the gains of controller, the matrix $R_{i 11}$ needs to guarantee $A_{i}+R_{i 11} K_{i}$ is stable. Therefore, we need to consider (4) for the input saturation in the region (6). However, for the sensor saturation, $R_{i 21}$ is chosen to be nonzero and based on the maximum value of $C_{i} x$ in the engineering practice $[24,26]$.

Lemma 8 (S-procedure). Let $Y_{0}(\eta), Y_{1}(\eta), \ldots, Y_{p}(\eta)$ be quadratic functions of $\eta \in R^{n}, Y_{i}(\eta)=\eta^{T} T_{i} \eta$, $i=0,1, \ldots, p$ with $T_{i}=T_{i}^{T}$. Then, the implication $Y_{1}(\eta) \leq 0, \ldots, Y_{p}(\eta) \leq 0 \Longrightarrow Y_{0}(\eta) \leq 0$ holds if there exist $\tau_{1}, \ldots, \tau_{p}>0$ such that

$$
T_{0}-\sum_{i=1}^{p} \tau_{i} T_{i} \leq 0
$$

Our aim in this paper is to solve the problem of composite control for the nonlinear saturating system (1) with multiple saturations and disturbances. Based on whether the system states are available, the full-order and reducedorder observers will be designed respectively. Here, the disturbance observer control approach will be applied for rejecting and attenuating the input disturbance, while the external disturbance will be attenuated by a $H_{\infty}$ control scheme.

\section{Main Result}

In this section, based on whether the system states are available, the composite full-order and reduced-order observerbased controllers are designed respectively. The input disturbance is rejected while external disturbance is attenuated.

3.1. Full-Order Observer-Based Controller. First of all, we suppose that the system states are not available and the nonlinearities $f_{i 1}(x), f_{i 2}(x)$ are known.
Defining the extended vector $\bar{x}(t)=\left[x^{T}(k), \omega^{T}(k)\right]^{T}$, together with (2) and (4), for $\sigma(k)=i$, given diagonal matrices $R_{i 11} \geq 0, R_{i 12} \geq 0, R_{i 21} \geq 0, R_{i 22} \geq 0$ satisfying $R_{i 12} \geq I>R_{i 11} \geq 0$ and $R_{i 22} \geq I>R_{i 21} \geq 0$, and any $\bar{u}(t) \in \mathscr{L}\left(R_{i 11} \bar{u}(k)\right)$, the system (1) can rewritten as follows:

$$
\begin{aligned}
\bar{x}(k+1)= & \bar{A}_{i} \bar{x}(k)+\bar{G}_{i 1} f(\bar{x}(k), k)+\bar{B}_{i} R_{i 11} u(k) \\
& +\bar{B}_{i} \Psi_{i 1}(k)+\bar{E}_{i 1} d(k) \\
y(k)= & \left(R_{i 21} \bar{C}_{i}+\bar{D}_{i}\right) \bar{x}(k)+\bar{G}_{i 2} f(\bar{x}(k), k) \\
& +\Psi_{i 2}(k)+\bar{E}_{2} d(k),
\end{aligned}
$$

where $f_{i 1}(\bar{x})=f_{i 1}(x), f_{i 2}(\bar{x})=f_{i 2}(x)$ and

$$
\begin{aligned}
f_{i}(\bar{x}(k), k) & =\left[\begin{array}{l}
f_{i 1}(\bar{x}) \\
f_{i 2}(\bar{x})
\end{array}\right], \\
d(k) & =\left[\begin{array}{l}
d_{2}(k) \\
d_{3}(k)
\end{array}\right], \\
\bar{A}_{i} & =\left[\begin{array}{cc}
A_{i} & B_{i} R_{i 11} V_{i} \\
0 & W_{i}
\end{array}\right], \\
\bar{B}_{i} & =\left[\begin{array}{cc}
B_{i} \\
0
\end{array}\right], \\
\bar{G}_{i 1} & =\left[\begin{array}{cc}
G_{i 1} & 0 \\
0 & 0
\end{array}\right], \\
\bar{E}_{i 1} & =\left[\begin{array}{cc}
E_{i 1} & 0 \\
0 & M_{i}
\end{array}\right], \\
\bar{C}_{i} & =\left[\begin{array}{ll}
C_{i} & 0
\end{array}\right], \\
\bar{D}_{i} & =\left[\begin{array}{ll}
0 & D_{i} V_{i}
\end{array}\right], \\
\bar{G}_{i 2} & =\left[\begin{array}{ll}
G_{i 2} & 0
\end{array}\right], \\
\bar{E}_{i 2} & =\left[\begin{array}{ll}
E_{i 2} & 0
\end{array}\right] .
\end{aligned}
$$

The full-order observer-based controller for the extended system (8) is designed as

$$
\begin{aligned}
\widehat{\bar{x}}(k+1)= & \bar{A}_{i} \hat{\bar{x}}(k)+\bar{B}_{i} R_{i 11} u(k)+\bar{G}_{i 1} f_{i}(\hat{\bar{x}}(k), k) \\
& +L_{i}(\widehat{y}(k)-y(k)) \\
\hat{y}(k)= & R_{i 21} \bar{C}_{i} \hat{\bar{x}}(k)+\bar{G}_{i 2} f_{i}(\hat{\bar{x}}(k), k) \\
& +\bar{D}_{i}(k) \hat{\bar{x}}(k) \\
u(k)= & K_{i} \widehat{x}(k)-\widehat{d}_{1}(k),
\end{aligned}
$$


where $f_{i 1}(\hat{\bar{x}})=f_{i 1}(\hat{x}), f_{i 2}(\hat{\bar{x}})=f_{2}(\hat{x})$ and

$$
\begin{aligned}
\hat{\bar{x}}(k) & =\left[\begin{array}{l}
\widehat{x}(k) \\
\widehat{\omega}(k)
\end{array}\right], \\
L_{i} & =\left[\begin{array}{l}
L_{i 1} \\
L_{i 2}
\end{array}\right], \\
f_{i}(\hat{\bar{x}}(k), k) & =\left[\begin{array}{l}
f_{i 1}(\hat{\bar{x}}) \\
f_{i 2}(\hat{\bar{x}})
\end{array}\right] .
\end{aligned}
$$

The estimation error is denoted as

$$
e(k)=\bar{x}(k)-\widehat{\bar{x}}(k)=\left[\begin{array}{l}
e_{x}(k) \\
e_{\omega}(k)
\end{array}\right]=\left[\begin{array}{c}
x(k)-\widehat{x}(k) \\
\omega(k)-\widehat{\omega}(k)
\end{array}\right],
$$

and then the error dynamic can be obtained

$$
\begin{aligned}
e(k+1)= & \left(\bar{A}_{i}-L_{i} R_{i 21} \bar{C}_{i}-L_{i} \bar{D}_{i}\right) e(k)+\bar{S}_{i} \Psi_{i}(k) \\
& +\left(\bar{E}_{i 1}-L_{i} \bar{E}_{i 2}\right) d(k) \\
& +\left(\bar{G}_{i 1}-L_{i} \bar{G}_{i 2}\right)\left(f_{i}(\bar{x})-f_{i}(\hat{\bar{x}})\right),
\end{aligned}
$$

where

$$
\begin{aligned}
\bar{S}_{i} & =\left[\begin{array}{ll}
\bar{B}_{i} & -L_{i}
\end{array}\right], \\
\Psi_{i}(k) & =\left[\begin{array}{l}
\Psi_{i 1}(k) \\
\Psi_{i 2}(k)
\end{array}\right] .
\end{aligned}
$$

Based on (10), (6) can be rewritten as

$$
\mathscr{L}\left(R_{i 11} \bar{u}(k)\right)=\left\{\left|\left(R_{i 11} \widetilde{K}_{i}\right)_{l} \tilde{x}\right| \leq 1, l \in \mathbf{I}[1, m]\right\},
$$

where

$$
\begin{aligned}
\widetilde{x}(k) & =\left[\begin{array}{l}
x(k) \\
e(k)
\end{array}\right], \\
\widetilde{K}_{i} & =\left[\begin{array}{lll}
K_{i} & -K_{i} & V_{i}
\end{array}\right] .
\end{aligned}
$$

For any $\widetilde{x}(k) \in \mathscr{L}\left(R_{i 11} \bar{u}(k)\right)$ with a given $R_{i 11}$, by (4), the closed-loop system can be described as

$$
\widetilde{x}(k+1)=\widetilde{A}_{i} \widetilde{x}(k)+\widetilde{G}_{i} \widetilde{f}_{i}(k)+\widetilde{E}_{i} d(k)+\widetilde{S}_{i} \Psi_{i}(k),
$$

where

$$
\begin{aligned}
& \widetilde{A}_{i}=\left[\begin{array}{cc}
A_{i}+B_{i} R_{i 11} K_{i} & B_{i} R_{i 11}\left[\begin{array}{cc}
-K_{i} & V_{i}
\end{array}\right] \\
0 & \bar{A}_{i}-L_{i} \bar{C}_{i}-L_{i} \bar{D}_{i}
\end{array}\right], \\
& \tilde{f}_{i}(k)=\left[\begin{array}{c}
f_{i 1}(x) \\
f_{i}(\bar{x})-f_{i}(\hat{\bar{x}})
\end{array}\right], \\
& \widetilde{S}_{i}=\left[\begin{array}{cc}
{\left[B_{i}\right.} & 0
\end{array}\right], \\
& \widetilde{G}_{i}=\left[\begin{array}{cc}
G_{i 1} & 0 \\
0 & \bar{G}_{i 1}-L_{i} \bar{G}_{i 2}
\end{array}\right] \text {, }
\end{aligned}
$$

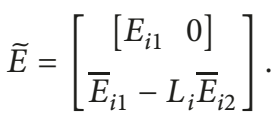

The reference output is chosen to be

$$
z(k)=\tilde{x}(k) .
$$

In the following theorem, we present some sufficient conditions under which a full-order observer-based controller can be designed to stabilize the closed-loop system (17) without external disturbances $d(k)$ and satisfy the $H_{\infty}$ performance requirement when the system is subject to external disturbances.

Theorem 9. For given diagonal matrices $R_{i 11} \geq 0, R_{i 12} \geq 0$ satisfying $R_{i 12} \geq I>R_{i 11} \geq 0$, diagonal matrices $R_{i 21} \geq$ $0, R_{i 22} \geq 0$ satisfying $R_{i 22} \geq I>R_{i 21} \geq 0$ and $\gamma>0$, under arbitrarily switching law, the closed-loop system (17) under the full-order state observer (10) for the augmented system (8) is robustly asymptotically stable in the absence of the disturbance $d(k)$ for all initial conditions $\Omega(P)=\left\{\tilde{x}^{T} P \tilde{x} \leq 1\right\}$ and satisfies the $H_{\infty}$ performance $\|z(k)\|_{2}<\gamma\|d(k)\|_{2}$, if there exist matrices $P_{1}>0, P_{2}>0, P_{3}>0, S_{1}>0, Y_{i 1}, Y_{i 2}, Y_{i 3}$ and parameters $\tau_{i}>0, i \in \mathbf{I}[1,5]$ such that

$$
P_{1} S_{1}=I .
$$

$$
\left[\begin{array}{cccc}
1 & \left(R_{i 11} Y_{i 1}\right)_{l} & -\left(R_{i 11} Y_{i 1}\right)_{l} & \left(R_{i 11} V_{i}\right)_{l} \\
* & P_{1} & 0 & 0 \\
* & * & P_{2} & 0 \\
* & * & * & P_{3}
\end{array}\right]>0,
$$

$l \in \mathbf{I}[1, m]$,

$$
\left[\begin{array}{cccc}
\Phi_{i 11} & \Phi_{i 12} & 0 & \Phi_{i 14} \\
* & \Phi_{i 22} & 0 & \Phi_{i 24} \\
* & * & -\gamma^{2} I & \Phi_{i 34} \\
* & * & * & \Phi_{i 44}
\end{array}\right]<0,
$$


where

$$
\begin{aligned}
& \breve{U}_{i 1}=\frac{\left(U_{i 11}^{T} U_{i 12}+U_{i 12}^{T} U_{i 11}\right)}{2}, \\
& \breve{U}_{i 2}=\frac{-\left(U_{i 11}^{T}+U_{i 12}^{T}\right)}{2} \\
& \breve{H}_{i 1}=\frac{\left(H_{i 11}^{T} H_{i 12}+H_{i 12}^{T} H_{i 11}\right)}{2}, \\
& \breve{H}_{i 2}=\frac{-\left(H_{i 11}^{T}+H_{i 12}^{T}\right)}{2}, \\
& \Theta_{i 1}=-P_{1}-\tau_{i 1} \breve{U}_{i 1}+I, \\
& \Theta_{i 2}=-P_{2}-\tau_{i 2} \breve{U}_{i 1}-\tau_{i 3} \breve{H}_{i 1}+I,
\end{aligned}
$$$$
\Phi_{i 11}=\left[\begin{array}{cccccc}
\Theta_{i 1} & 0 & 0 & -\tau_{i 1} \breve{U}_{i 2} & 0 & 0 \\
0 & \Theta_{i 2} & 0 & 0 & -\tau_{i 2} \breve{U}_{i 2} & -\tau_{i 3} \breve{H}_{i 2} \\
0 & 0 & -P_{3}+I & 0 & 0 & 0 \\
-\tau_{i 1} \breve{U}_{i 2}^{T} & 0 & 0 & -\tau_{i 1} I & 0 & 0 \\
0 & -\tau_{i 2} \breve{U}_{i 2}^{T} & 0 & 0 & -\tau_{i 2} I & 0 \\
0 & -\tau_{i 3} \breve{H}_{i 2}^{T} & 0 & 0 & 0 & -\tau_{i 3} I
\end{array}\right] \text {, }
$$$$
\Phi_{i 12}^{T}=\left[\begin{array}{cccccc}
R_{i 1} Y_{i 1} & -R_{i 1} Y_{i 1} & R_{i 1} V_{i} & 0 & 0 & 0 \\
\tau_{i 5} R_{i 2} C_{i} & 0 & 0 & 0 & 0 & 0
\end{array}\right] \text {, }
$$$$
\Phi_{i 14}
$$$$
=\left[\begin{array}{ccc}
A_{i}^{T}+Y_{i 1}^{T} R_{i 1} B_{i}^{T} & 0 & 0 \\
-Y_{i 1}^{T} R_{i 11} B_{i}^{T} & A_{i}^{T} P_{2}-C_{i}^{T} Y_{i 2}^{T} & C_{i}^{T} Y_{i 3}^{T} \\
V_{i}^{T} R_{i 11} B_{i}^{T} & V_{i}^{T} R_{i 11} B_{i}^{T} P_{2}^{T}-V_{i} D_{i}^{T} Y_{i 2}^{T} & W_{i} P_{3}+V_{i} D_{i}^{T} Y_{i 3}^{T} \\
G_{i 1}^{T} & 0 & 0 \\
0 & G_{i 1}^{T} P_{2}-G_{i 2}^{T} Y_{i 2}^{T} & -G_{i 2}^{T} Y_{i 3}^{T} \\
0 & 0 & 0
\end{array}\right],
$$$$
\Phi_{i 22}=\left[\begin{array}{cc}
-2 \tau_{i 4} I & 0 \\
0 & -2 \tau_{i 5} I
\end{array}\right] \text {, }
$$$$
\Phi_{i 24}=\left[\begin{array}{ccc}
B_{i}^{T} & B_{i}^{T} P_{2} & 0 \\
0 & -Y_{i 2}^{T} & -Y_{i 3}^{T}
\end{array}\right] \text {, }
$$$$
\Phi_{i 34}^{T}=\left[\begin{array}{cc}
E_{i 1} & 0 \\
P_{2} E_{i 1}-Y_{i 1} E_{i 2} & 0 \\
0 & P_{3} M_{i}-Y_{i 3} E_{i 2}
\end{array}\right] \text {, }
$$$$
\Phi_{i 44}=\left[\begin{array}{ccc}
-S_{1} & 0 & 0 \\
0 & -P_{2} & 0 \\
0 & 0 & -P_{3}
\end{array}\right] \text {. }
$$

Moreover, if the above conditions are feasible, the gains of the desired observer and controller are given by

$$
\begin{aligned}
K_{i} & =Y_{i 1}, \\
L_{i 1} & =P_{2}^{-1} Y_{i 2}, \\
L_{i 2} & =P_{3}^{-1} Y_{i 3} .
\end{aligned}
$$

Proof. Choose the common Lyapunov candidate as follows:

$$
V(\tilde{x}(k))=\tilde{x}^{T}(k) P \tilde{x}(k),
$$

where $P=\operatorname{diag}\left\{P_{1}, P_{2}, P_{3}\right\}$ with $P_{j}>0, j=1,2,3$.
For any $\tilde{x}(k) \in \mathscr{L}\left(R_{i 11} \bar{u}(t)\right)$, along the closed-loop augmented system (17) with $d(k) \equiv 0$, the difference of $V(k)$ can be calculated as

$$
\begin{aligned}
\Delta V(\tilde{x}(k)) & =\tilde{x}^{T}(k+1) P \tilde{x}(k+1)-\tilde{x}^{T}(k) P \tilde{x}(k) \\
& =\eta(k)^{T}\left[\mathscr{A}_{i 0}^{T} P \mathscr{A}_{i 0}-\mathscr{P}\right] \eta(k),
\end{aligned}
$$

where

$$
\begin{aligned}
\eta^{T}(k) & =\left[\begin{array}{lll}
\widetilde{x}^{T}(k) & \widetilde{f}_{i}^{T}(k), \Psi_{i}^{T}(k)
\end{array}\right], \\
\mathscr{A}_{0 i} & =\left[\begin{array}{lll}
\widetilde{A}_{i} & \widetilde{G}_{i} & \widetilde{S}_{i}
\end{array}\right], \\
\mathscr{P} & =\operatorname{diag}\left\{\begin{array}{lll}
P, & 0, & 0
\end{array}\right\} .
\end{aligned}
$$

From the assumption that the nonlinearities $f_{1}(k)$ and $f_{2}(k)$ belong to sectors $\left[U_{i 11}, U_{i 12}\right]$ and $\left[H_{i 21}, H_{i 22}\right]$, it can be verified that

$$
\begin{aligned}
& {\left[\begin{array}{c}
x(k) \\
f_{i 1}(x)
\end{array}\right]^{T}\left[\begin{array}{cc}
\breve{U}_{i 1} & \breve{U}_{i 2} \\
* & I
\end{array}\right]\left[\begin{array}{c}
x(k) \\
f_{i 1}(x)
\end{array}\right] \leq 0,} \\
& {\left[\begin{array}{c}
e_{x}(k) \\
f_{i 1}(\bar{x})-f_{i 1}(\hat{\bar{x}})
\end{array}\right]^{T}\left[\begin{array}{cc}
\breve{U}_{i 1} & \breve{U}_{i 2} \\
* & I
\end{array}\right]\left[\begin{array}{c}
e_{x}(k) \\
f_{i 2}(\bar{x})-f_{i 2}(\widehat{\bar{x}})
\end{array}\right]}
\end{aligned}
$$$$
\leq 0
$$

$$
\begin{aligned}
& {\left[\begin{array}{c}
e_{x}(k) \\
f_{i 2}(\bar{x})-f_{i 2}(\hat{\bar{x}})
\end{array}\right]^{T}\left[\begin{array}{cc}
\breve{H}_{i 1} & \breve{H}_{i 2} \\
* & I
\end{array}\right]\left[\begin{array}{c}
e_{x}(k) \\
f_{i 2}(\bar{x})-f_{i 2}(\widehat{\bar{x}})
\end{array}\right]} \\
& \quad \leq 0
\end{aligned}
$$

where $\breve{U}_{i 1}, \breve{U}_{i 2}, \breve{H}_{i 1}$, and $\breve{H}_{i 2}$ are defined in (1).

From (22), the following inequality can be obtained:

$$
\left[\begin{array}{ccc}
\widetilde{\Phi}_{i 11} & \Phi_{i 12} & \Phi_{i 14} \\
* & \Phi_{i 22} & \Phi_{i 24} \\
* & * & \Phi_{i 44}
\end{array}\right]<0,
$$

where $\widetilde{\Phi}_{i 11}=\Phi_{i 11}-\operatorname{diag}\{I I, I, 0,0,0\}$.

Noting the facts of $Y_{i 1}=K_{i}, Y_{i 2}=P_{2} L_{i 1}$, and $Y_{i 3}=P_{3} L_{i 2}$, before and after multiplying (29) by $\operatorname{diag}\left\{I \tau_{i 4}^{-1}, I, P_{1}, I\right\}$ and by the condition (20), it yields that

$$
\left[\begin{array}{ccc}
\widetilde{\Phi}_{i 11} & \Phi_{i 12} & \widetilde{\Phi}_{i 14} \\
* & \Phi_{i 22} & \widetilde{\Phi}_{i 24} \\
* & * & P
\end{array}\right]<0,
$$

where $\widetilde{\Phi}_{i 14}=\Phi_{i 14} \widetilde{P}_{1}$ and $\widetilde{\Phi}_{i 24}=\Phi_{i 24} \widetilde{P}_{1}$ with $\widetilde{P}_{1}=$ $\operatorname{diag}\left\{P_{1}, I, I\right\}$.

Together (5), (28) with (30), using Lemma 8 and Schur complement, we can get

$$
\begin{aligned}
\Delta V(\tilde{x}(k)) & <0, \\
\tilde{x}(k) & \in \mathscr{L}\left(R_{i 11} \bar{u}(t)\right) .
\end{aligned}
$$


Using Schur complement, (21) is equivalent to

$$
\left(R_{i 11} \widetilde{K}\right)_{l} P^{-1}\left(R_{i 11} \widetilde{K}\right)_{l}^{T} \leq 1,
$$

which means that

$$
\Omega(P) \subset \mathscr{L}\left(R_{i 11} \widetilde{K}_{i} \tilde{x}(k)\right) .
$$

From (30) and (33), we know that the augmented closed system (17) without external disturbance $d(k)$ is asymptotically stable with an initial set $\Omega(P)$.

Next, we suppose that system (17) is subject to external disturbances $d(k) \neq 0$.

For given $\gamma>0$, consider the index as follows:

$$
J(N)=\sum_{k=1}^{N}\left(z^{T}(k) z(k)-\gamma^{2} d^{T}(k) d(k)\right) .
$$

Under the zero initial condition, from (25), we have

$$
J(N) \leq \sum_{k=1}^{N}\left(z^{T}(k) z(k)-\gamma^{2} d^{T}(k) d(k)+\Delta V(k)\right)
$$

From the above discussion and conditions (20), (22), we can readily derive

$$
J(N) \leq 0,
$$

and then, for nonzero $d(k) \in \mathscr{L}(0, \infty)$, one has

$$
\lim _{N \rightarrow \infty} J(N) \leq 0
$$

which means that the $H_{\infty}$ performance $\|z(k)\|_{2} \leq \gamma\|d(k)\|_{2}$ is satisfied.

3.2. Reduced-Order Observer-Based Controller. Assuming that the states of the system (1) are available, a disturbance observer can be formulated as

$$
\begin{aligned}
\widehat{d}_{1}(k) & =V_{\sigma(k)} \widehat{\omega}(k) \\
\widehat{\omega}(k) & =v(k)-L_{\sigma(k)} x(k),
\end{aligned}
$$

where $v(k)$ is generated by the following dynamic:

$$
\begin{aligned}
& v(k+1)=\left(W_{\sigma(k)}+L_{\sigma(k)} B_{\sigma(k)} R_{\sigma(k), 11} V_{\sigma(k)}\right)(v(k) \\
& \left.-L_{\sigma(k)} x(k)\right)+L_{\sigma(k)}\left(A_{\sigma(k)} x(t)\right. \\
& \left.+B_{\sigma(k)} R_{\sigma(k), 11} u(k)+G_{\sigma(k), 1} f_{\sigma(k), 1}(x(k), k)\right) .
\end{aligned}
$$

Then, the dynamic of the estimation of disturbance error $e_{\omega}(k)$ is

$$
\begin{aligned}
e_{\omega}(k+1)= & \left(W_{\sigma(k)}+L_{\sigma(k)} B_{\sigma(k)} R_{\sigma(k), 11} V\right) e_{\omega}(k) \\
& +L_{\sigma(k)} B_{\sigma(k)} \Psi_{\sigma(k), 1}(k)+M_{\sigma(k)} d_{3}(k) \\
& +L_{\sigma(k)} E_{\sigma(k), 1} d_{2}(k) .
\end{aligned}
$$

A disturbance observer-based controller is designed as

$$
u(k)=K_{\sigma(k)} x(k)-\widehat{d}_{1}(k) .
$$

Combining (1), (38), and (40), for $\sigma(k)=i \in \mathbf{I}[1, q]$, the closed-loop system for the extended state $\mathbb{x}(k)=$ $\left[\begin{array}{ll}x^{T}(k) & e_{\omega}^{T}(k)\end{array}\right]^{T}$ can be described as

$$
\mathbb{x}(k+1)=\mathbb{A}_{i} \mathbb{x}(k)+\mathbb{G}_{i} f_{i}(k)+\mathbb{S}_{i} \Psi_{1}(k)+\mathbb{E}_{i} d(k),
$$

where

$$
\begin{aligned}
& \mathbb{A}_{i}=\left[\begin{array}{cc}
A_{i}+B_{i} R_{i 11} K_{i} & B_{i} R_{i 11} V_{i} \\
0 & W_{i}+L_{i} B_{i} R_{i 11} V
\end{array}\right], \\
& \mathbb{S}_{i}=\left[\begin{array}{c}
B_{i} \\
L_{i} B_{i}
\end{array}\right], \\
& \mathbb{G}_{i}=\left[\begin{array}{c}
G_{i 1} \\
0
\end{array}\right], \\
& \mathbb{E}_{i}=\left[\begin{array}{cc}
E_{i 1} & 0 \\
L_{i} E_{i 1} & M_{i}
\end{array}\right] .
\end{aligned}
$$

For this case, (6) can be rewritten as

$$
\mathscr{L}\left(R_{i 11} \bar{u}(k)\right)=\left\{\left|\left(R_{i 11} \mathbb{K}_{i}\right) \mathbb{x}\right|_{l} \leq 1, l \in \mathbf{I}[1, m]\right\},
$$

where $\mathbb{K}_{i}=\left[K_{i}, V_{i}\right]$. The reference output is set as

$$
\mathbb{Z}(k)=\mathbb{x}(k),
$$

Theorem 10. Given diagonal matrices $R_{i 11} \geq 0, R_{i 12} \geq 0$ satisfying $R_{i 12} \geq I>R_{i 11} \geq 0$ and $\gamma>0$, under arbitrarily switching law, the augmented system (42) is robustly asymptotically stable in the absence of the disturbance $d(k)$ for all initial conditions $\Omega(P)=\left\{\tilde{x}^{T} P \tilde{x} \leq 1\right\}, P=\operatorname{diag}\left\{P_{1}, P_{2}\right\}$, and satisfies the $H_{\infty}$ performance $\|z(k)\|_{2}<\gamma\|d(k)\|_{2}$, if there exist matrices $P_{1}>0, P_{2}>0, S_{1}>0, Y_{i 1}, Y_{i 2}$ and parameters $\tau_{i}>0, i \in \mathbf{I}[1,5]$ such that

$$
P_{1} S_{1}=I \text {, }
$$

$$
\begin{gathered}
{\left[\begin{array}{ccc}
1 & \left(R_{i 11} Y_{i 1}\right)_{l} & \left(R_{i 11} V_{i}\right)_{l} \\
* & P_{1} & 0 \\
* & * & P_{2}
\end{array}\right]>00, \quad l \in \mathbf{I}[1, m],} \\
{\left[\begin{array}{ccc}
\Psi_{i 11} & 0 & \Psi_{i 13} \\
* & -\gamma^{2} I & \Psi_{i 23} \\
* & * & \Psi_{i 33}
\end{array}\right]<0,}
\end{gathered}
$$


where $\breve{U}_{i 1}$ and $\breve{U}_{i 2}$ were defined in (1), and

$$
\begin{aligned}
& \Psi_{i 11}=\left[\begin{array}{cccc}
-P_{1}-\tau_{i 1} \breve{U}_{i 1}+I & 0 & -\tau_{i 1} \breve{U}_{i 2} & Y_{i 1}^{T} R_{i 1} \\
0 & -P_{2}+I & 0 & V_{i}^{T} R_{i 1} \\
-\tau_{i 1} \breve{U}_{i 2}^{T} & 0 & -\tau_{i 1} I & 0 \\
R_{i 1} Y_{i 1} & R_{i 1} V_{i} & 0 & -2 \tau_{i 2} I
\end{array}\right], \\
& \Psi_{i 13}^{T} \\
& =\left[\begin{array}{cccc}
A_{i}+B_{i} R_{i 11} Y_{i 1} & B_{i} R_{i 1} V_{i} & G_{i 1} & B_{i} \\
0 & P_{2} W_{i}+Y_{i 2} B_{i} R_{i 1} V_{i} & 0 & Y_{i 2} B_{i}
\end{array}\right], \\
& \Psi_{i 23}=\left[\begin{array}{cc}
E_{i 1}^{T} & E_{i 1}^{T} Y_{i 2}^{T} \\
0 & M_{i}^{T} P_{2}
\end{array}\right], \\
& \Psi_{i 33}=\left[\begin{array}{cc}
-S_{1} & 0 \\
0 & -P_{2}
\end{array}\right] .
\end{aligned}
$$

Moreover, if the above conditions are feasible, the gains of the desired observer and controller are given by

$$
\begin{aligned}
& K_{i}=Y_{i 1}, \\
& L_{i}=P_{2}^{-1} Y_{i 2} .
\end{aligned}
$$

Proof. A Lyapunov candidate function is chosen as

$$
V(\mathbb{x}(t))=\nwarrow^{T}(t) P_{\rtimes}(t),
$$

where $P=\operatorname{diag}\left\{P_{1}, P_{2}\right\}$ with $P_{i}>0, i=1,2$.

For any $\mathbb{x}(k) \in \mathscr{L}\left(R_{i 11} \mathbb{K}_{i} \rtimes(k)\right)$, the difference of $V(k)$ along system (42) in the absence of disturbance $d(k)$ is

$$
\begin{aligned}
\Delta V(k) & =\nwarrow^{T}(k+1) P \rtimes(k+1)-\nwarrow(k)^{T} P_{\nwarrow}(k) \\
& =\Theta^{T}(k)\left[\mathbb{A}_{0 i}^{T} P \mathbb{A}_{0 i}-\mathbb{P}\right] \Theta(k),
\end{aligned}
$$

where

$$
\begin{aligned}
\Theta(k) & =\left[\begin{array}{lll}
\mathbb{x}(k) & f_{i}(k) & \Psi_{i}(k)
\end{array}\right], \\
\mathbb{A}_{0 i} & =\left[\begin{array}{lll}
\mathbb{A}_{i} & \mathbb{G}_{i} & \mathbb{S}_{i}
\end{array}\right], \\
\mathbb{P} & =\operatorname{diag}\left\{\begin{array}{lll}
P, & 0, & 0
\end{array}\right\} .
\end{aligned}
$$

Defining $Y_{i 1} \triangleq q K_{i}$ and $Y_{i 2} \triangleq q P_{2} L_{i}$ and performing a congruence transformation to (48) by $\operatorname{diag}\left\{I, I, P_{1}, I\right\},(48)$ is equivalent to

$$
\left[\begin{array}{ccc}
\Psi_{i 11} & 0 & \bar{\Psi}_{i 13} \\
* & -\gamma^{2} I & \bar{\Psi}_{i 23} \\
* & * & \bar{\Psi}_{i 33}
\end{array}\right]<0,
$$

where

$$
\begin{aligned}
& \bar{\Psi}_{i 13}^{T} \\
& =\left[\begin{array}{cccc}
P_{1} A_{i}+P_{1} B_{i} R_{i 1} Y_{i 1} & P_{1} B_{i} R_{i 11} V_{i} & P_{1} G_{i 1} & P_{1} B_{i} \\
0 & P_{2} W_{i}+P_{2} L_{i} B_{i} R_{i 11} V_{i} & 0 & P_{2} L_{i} B_{i}
\end{array}\right], \\
& \bar{\Psi}_{i 23}^{T}=\left[\begin{array}{cc}
P_{1} E_{i 1} & 0 \\
P_{2} L_{i} E_{i 1} & P_{2} M_{i}
\end{array}\right] \text {, } \\
& \bar{\Psi}_{i 33}=\left[\begin{array}{cc}
-P_{1} & 0 \\
0 & -P_{2}
\end{array}\right] \text {. }
\end{aligned}
$$

Equation (56) yields that

$$
\begin{gathered}
\Delta V(k)-\tau_{i 1}\left(f_{i 1}(k)-U_{i 11} x\right)\left(f_{i 1}(k)-U_{i 12} x\right) \\
-2 \tau_{i 2} \Phi_{i 1}^{T}(k)\left(\Phi_{i 1}-R_{i 1} \mathbb{k} \ll(k)\right) \leq 0
\end{gathered}
$$

Based on the assumption that $f_{i 1}$ belongs to the sector $\left[U_{i 11}, U_{i 12}\right]$ and (28), by Lemma 8, we have

$$
\Delta V(k) \leq 0 .
$$

Under the conditions of zero initial state and nonzero disturbance $d(k)$, defining the $H_{\infty}$ performance index for given $\gamma>0$ as

$$
J(N)=\sum_{k=1}^{N}\left(\mathbb{Z}^{T}(k) \mathbb{z}(k)-\gamma^{2} d^{T}(k) d(k)\right) .
$$

By Schur complement to (56) and (47), along the same line in the proof of Theorem 9, we can represent that

$$
\|\mathbb{Z}\|_{2} \leq \gamma\|d(k)\|, \quad \text { for } d(k) \in \mathscr{L}[0, \infty),
$$

and

$$
\Omega(P) \subset \mathscr{L}\left(R_{i 11} \mathbb{K} \mathbb{x}(k)\right) .
$$

From (57), (59), and (60), the desired results are obtained and the proof is completed.

Remark 11. The composite antidisturbance control problem has been solved by designing disturbance observer-based controller for the switched system (1) subject to input saturation and multiple disturbances. The disturbance observerbased control method is used to reject the input path disturbances generated by system (2), and for the external disturbances which belong to $\mathscr{L}[0, \infty)$, the attenuation performance are achieved by $H_{\infty}$ control method. The most existing results about composite antidisturbance control do not consider the switched systems subject to saturation, such as actuator saturation and sensor saturation. We solved the problem from comprehensive perspective and gave the results from both full-order and reduced-order observerbased composite control methods.

Remark 12. Although, due to (20) and (46), the conditions in Theorems 9 and 10 are not rigid constraints in the framework of linear matrix inequalities (LMIs), here, the following algorithms are proposed to this nonconvex feasibility problem. 
Algorithm 13.

Step 1 (initialization). Choose the appropriate initial positive definite diagonal matrices $R_{i 11}, R_{i 12}, R_{i 21}, R_{i 22}$ satisfying $R_{i 12} \geq I>R_{i 11} \geq 0$ and $R_{i 22} \geq I>R_{i 21} \geq 0$, scalar $\lambda>0$, and find a feasible point $M_{k}, N_{k}$. If there are none, exit. Set $k=0$.

Step 2. Solve matrices $P_{1}, S_{1}$ by the following problem:

$$
\begin{array}{ll}
\text { Minimize } & \operatorname{TR}\left\{M_{k} P_{1}+N_{k} S_{1}\right\} \\
\text { subject to } & \text { (a) }\left[\begin{array}{cc}
P_{1} & I \\
I & S_{1}
\end{array}\right] \geq 0, \\
& \text { (b) inequalities (21) and (22) hold. }
\end{array}
$$

Step 3. Set $M_{k+1}=S_{1}$ and $N_{k+1}=P_{1}$. If $\operatorname{TR}\left\{M_{k+1} N_{k+1}\right\}=n$, stop the iteration; otherwise, go back to Step 2 .

\section{Algorithm 14.}

Step 1 (initialization). Choose the appropriate initial positive definite diagonal matrices $R_{i 11}, R_{i 12}$ satisfying $R_{i 12} \geq I>$ $R_{i 11} \geq 0$ and scalar $\lambda>0$, and find a feasible point $M_{k}, N_{k}$. If there are none, exit. Set $k=0$.
Step 2. Solve matrices $P_{1}, S_{1}$ by the following problem:

$$
\begin{array}{ll}
\text { Minimize } & \operatorname{TR}\left\{M_{k} P_{1}+N_{k} S_{1}\right\} \\
\text { subject to } & (a)\left[\begin{array}{cc}
P_{1} & I \\
I & S_{1}
\end{array}\right] \geq 0,
\end{array}
$$

(b) inequalities (47) and (48) hold.

Step 3. Set $M_{k+1}=S_{1}$ and $N_{k+1}=P_{1}$. If $\operatorname{TR}\left\{M_{k+1} N_{k+1}\right\}=n$, stop the iteration; otherwise, go back to Step 2 .

Remark 15. With the help of the Algorithms 13 and 14, we will propose further the following algorithms to optimize the design schemes of the controllers for estimation of the domain of initial states as large as possible.

\section{Algorithm 16.}

Step 1. Choose the initial scalar $\gamma>0$, the appropriate matrix $R>0$, the initial matrices $P_{1}>0$ and $S_{1}>0$ which are obtained from Algorithm 13.

Step 2. Use the initialization from Step 1 to solve the following problem for $\alpha$ :

$$
\begin{aligned}
\operatorname{Inf}_{P_{2}>0, P_{3}>0, \tau_{i}>0, i=1,2,3,4,5, Y_{i j}, j=1,2,3} & \alpha \\
\text { s.t. } & \text { (a) } P-\alpha R \leq 0, \text { where } P=\operatorname{diag}\left\{P_{1}, P_{2}, P_{3}\right\} ; \\
& \text { (b) inequalities (21) and (22) hold. }
\end{aligned}
$$

\section{Algorithm 17.}

Step 1. Choose the initial scalar $\gamma>0$, the appropriate matrix $R>0$, the initial matrices $P_{1}>0$ and $S_{1}>0$ which are obtained from Algorithm 13.

Step 2. Use the initialization from Step 1 to solve the following problem for $\alpha$ :

$$
\begin{aligned}
\operatorname{Inf}_{P_{2}>0, Y_{i j}, j=1,2} & \alpha \\
\text { s.t. } & \text { (a) } P-\alpha R \leq 0,
\end{aligned}
$$

$$
\text { where } P=\operatorname{diag}\left\{P_{1}, P_{2}\right\} \text {; }
$$

(b) inequalities (47) and (48) hold.

Remark 18. The results of both Theorems 9 and 10 are established under the arbitrary switching rules due to the common Lyapunov function being chosen, and how to choose less conservative Lyapunov function is one of our future works. When the switching rules $\sigma(k) \equiv i$, that means the switched system always stays in the $i_{t h}$ subsystem, the result can be used for each subsystem as a special and independent case.
Assumption 19. The nonlinearity $f_{i 1}(x(k), k)$ satisfies the Lipschitz condition, namely, $f_{i 1}(0,0)=0$ and

$$
\begin{aligned}
& \left\|f_{i 1}(x(k), k)-f_{i 1}(y(k), k)-U_{i 11}(x(k)-y(k))\right\| \\
& \quad \leq 0 .
\end{aligned}
$$

Corollary 20. Given diagonal matrices $R_{i 11} \geq 0, R_{i 12} \geq 0$ satisfying $R_{i 12} \geq I>R_{i 11} \geq 0$ and $\gamma>0$, under Assumption 19 and arbitrarily switching law, the augmented system (42) is robustly asymptotically stable in the absence of the disturbance $d(k)$ for all initial conditions $\Omega(P)=\left\{\tilde{x}^{T} P \tilde{x} \leq 1\right\}, P=$ $\operatorname{diag}\left\{P_{1}, P_{2}\right\}$, and satisfies the $H_{\infty}$ performance $\|z(k)\|_{2}<$ $\gamma\|d(k)\|_{2}$, if there exist matrices $X_{1}>0, P_{2}>0, Y_{i 1}, Y_{i 2}$ and parameters $\tau_{i}>0, i \in \boldsymbol{I}[1,5]$ such that

$$
\begin{aligned}
& {\left[\begin{array}{ccc}
1 & \left(R_{i 11} Y_{i 1}\right)_{l} & \left(R_{i 11} V_{i}\right)_{l} \\
* & -X_{1} & 0 \\
* & * & P_{2}
\end{array}\right]<0, \quad l \in \boldsymbol{I}[1, m]} \\
& \\
& {\left[\begin{array}{ccc}
\Lambda_{i 11} & 0 & \Lambda_{i 13} \\
* & -\gamma^{2} I & \Lambda_{i 23} \\
* & * & \Lambda_{i 33}
\end{array}\right]<0,}
\end{aligned}
$$


where $\breve{U}_{i 1}$ and $\breve{U}_{i 2}$ were defined in (1), and

$$
\begin{aligned}
& \Lambda_{i 11}=\left[\begin{array}{cccc}
-X_{1} & 0 & -\tau_{i 1} X_{1} \breve{U}_{i 11} & Y_{i 1}^{T} R_{i 1} \\
0 & -P_{2}+I & 0 & V_{i}^{T} R_{i 1} \\
-\tau_{i 1} \breve{U}_{i 11}^{T} X_{1} & 0 & -\tau_{i 1} I & 0 \\
R_{i 1} Y_{i 1} & R_{i 1} V_{i} & 0 & -2 \tau_{i 2} I
\end{array}\right], \\
& \Lambda_{i 13}^{T} \\
& =\left[\begin{array}{cccc}
A_{i} X_{1}+B_{i} R_{i 11} Y_{i 1} & B_{i} R_{i 11} V_{i} & G_{i 1} & B_{i} \\
0 & W_{i}+L_{i} B_{i} R_{i 11} V_{i} & 0 & L_{i} B_{i} \\
X_{1} & 0 & 0 & 0
\end{array}\right], \\
& \Lambda_{i 23}=\left[\begin{array}{ccc}
E_{i 1}^{T} & E_{i 1}^{T} L_{i}^{T} & 0 \\
0 & M_{i}^{T} & 0
\end{array}\right], \\
& \Lambda_{i 33}= \\
& 0
\end{aligned}
$$

Moreover, if the above conditions are feasible, the gains of the desired observer and controller are given by

$$
\begin{aligned}
& P_{1}=X_{1}^{-1}, \\
& K_{i}=Y_{i 1} P_{1}, \\
& L_{i}=Y_{i 2} .
\end{aligned}
$$

Proof. Setting the notation (69), performing a congruence transformation to (66) by $\operatorname{diag}\left\{I P_{1}, I\right\}$ and to (67) by $\operatorname{diag}\left\{P_{1}, I, I, I, I, I, I, I, I\right\}$ and using Schur complement, from the fact $U_{i 11}^{T} U_{i 11} \geq 0$, one has (21) and (56), under Assumption 19, and the proof follows Theorem 10.

Remark 21. The nonlinearities $f_{i 1}$ under Assumption 19 are investigated widely in the most of results of the composite antidisturbance control [36]. The result of Corollary 20 is convex due to being in the framework of linear matrix inequalities (LMIs); therefore, it is feasible.

Remark 22. In the above discussion, the nonlinearities $f_{i 1}$ and $f_{i 2}$ are assumed to be known. The results about the case of the unknown nonlinearities are easy to be obtained by removing the information of $f_{i 1}$ and $f_{i 2}$ in the observers (10) and (40); the details are omitted here for space saving.

\section{Simulation Example}

In this section, system (1) with two subsystems is presented to illustrate the effectiveness of the proposed control design schemes, the parameters of which are shown as

$$
\begin{aligned}
\text { Subsystem 1: } & \mathrm{A}_{1}=\left[\begin{array}{ll}
0.1 & 0.2 \\
0.2 & 0.3
\end{array}\right], \\
B_{1} & =\left[\begin{array}{l}
0.2 \\
0.1
\end{array}\right],
\end{aligned}
$$

$$
\begin{aligned}
& G_{1,1}=\left[\begin{array}{l}
0.1 \\
0.2
\end{array}\right], \\
& E_{1,1}=\left[\begin{array}{l}
0.2 \\
0.1
\end{array}\right], \\
& C_{1}^{T}=\left[\begin{array}{l}
0.1 \\
0.1
\end{array}\right], \\
& D_{1}=\left[\begin{array}{l}
0.1 \\
]
\end{array},\right. \\
& G_{1,2}=\left[\begin{array}{l}
0.1
\end{array}\right], \\
& E_{1,2}=\left[\begin{array}{l}
0.1
\end{array}\right], \\
& W_{1}=\left[\begin{array}{ll}
0.2 & 0.1 \\
0.4 & 0.5
\end{array}\right], \\
& M_{1}=\left[\begin{array}{l}
0.1 \\
0.4
\end{array}\right], \\
& V_{1}=\left[\begin{array}{ll}
0.2 & 0.2
\end{array}\right],
\end{aligned}
$$

Subsystem 2: $\quad \mathrm{A}_{2}=\left[\begin{array}{ll}0.2 & 0.1 \\ 0.5 & 0.1\end{array}\right]$,

$$
B_{2}=\left[\begin{array}{l}
0.6 \\
0.4
\end{array}\right] \text {, }
$$$$
G_{2,1}=\left[\begin{array}{l}
0.1 \\
0.1
\end{array}\right] \text {, }
$$$$
E_{2,1}=\left[\begin{array}{l}
0.5 \\
0.1
\end{array}\right] \text {, }
$$$$
C_{2}^{T}=\left[\begin{array}{l}
0.1 \\
0.1
\end{array}\right] \text {, }
$$$$
D_{2}=[0.1] \text {, }
$$$$
G_{2,2}=[0.2] \text {, }
$$$$
E_{2,2}=[0.1] \text {, }
$$$$
W_{2}=\left[\begin{array}{ll}
0.1 & 0.2 \\
0.2 & 0.1
\end{array}\right],
$$$$
M_{2}=\left[\begin{array}{l}
0.1 \\
0.4
\end{array}\right] \text {, }
$$$$
V_{2}=\left[\begin{array}{ll}
0.1 & 0.1
\end{array}\right] \text {. }
$$

Setting $R_{i 11}=0.05, R_{i 12}=0.1$ for $i=1$, , by utilizing the proposed Algorithms 13 and 16, we can get $a=0.5919$ and

$$
P_{1}=\left[\begin{array}{ll}
1.4012 & 0.0230 \\
0.0230 & 1.4012
\end{array}\right],
$$




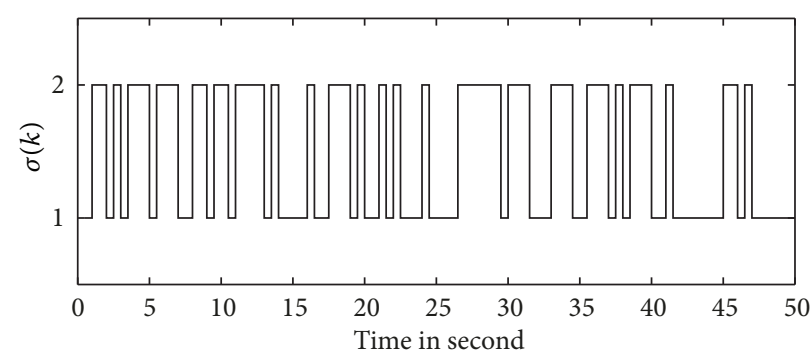

FIGURE 1: Switching signal.

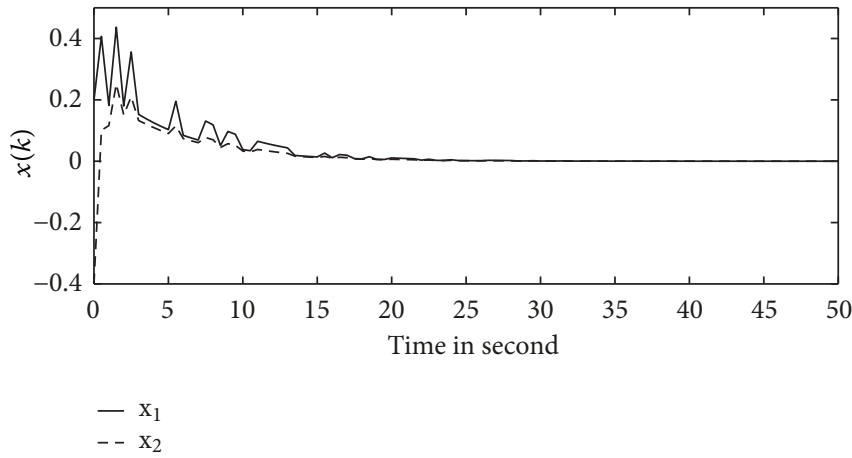

FIGURE 2: The state trajectories of System (1) under full-order observer-based controller (10).

$$
\begin{aligned}
P_{2} & =\left[\begin{array}{cc}
1.8336 & -0.5010 \\
-0.5010 & 1.7315
\end{array}\right], \\
P_{3} & =\left[\begin{array}{ll}
1.8960 & 0.1413 \\
0.1413 & 2.1901
\end{array}\right], \\
S_{1} & =\left[\begin{array}{ll}
0.7139 & -0.0117 \\
-0.0117 & 0.7139
\end{array}\right], \\
K_{11}^{T} & =\left[\begin{array}{l}
0.2802 \\
0.2962
\end{array}\right], \\
K_{21}^{T} & =\left[\begin{array}{l}
-1.9447 \\
-0.1290
\end{array}\right], \\
L_{11} & =\left[\begin{array}{l}
0.3791 \\
0.5463
\end{array}\right], \\
L_{21} & =\left[\begin{array}{l}
1.6554 \\
1.4216
\end{array}\right], \\
L_{12} & =\left[\begin{array}{l}
0.3404 \\
0.5321
\end{array}\right], \\
L_{22} & =\left[\begin{array}{l}
1.1656 \\
1.0207
\end{array}\right] ;
\end{aligned}
$$

$$
\begin{aligned}
P_{2} & =\left[\begin{array}{ll}
1.9765 & 0.4729 \\
0.4729 & 1.9576
\end{array}\right], \\
S_{1} & =\left[\begin{array}{cc}
0.6478 & -0.1075 \\
-0.1075 & 0.6478
\end{array}\right], \\
K_{21}^{T} & =\left[\begin{array}{c}
-4.0106 \\
0.4136
\end{array}\right], \\
L_{11} & =\left[\begin{array}{ll}
-0.0007 & -0.0003 \\
-0.0155 & -0.0077
\end{array}\right], \\
L_{21} & =10^{-3} *\left[\begin{array}{ll}
-0.4665 & -0.1546 \\
-0.4527 & -0.1840
\end{array}\right] .
\end{aligned}
$$

With $f_{i 1}(x(k))=\sin \left(0.1 * x_{1}(k)\right), f_{i 2}(x(k))=0, d_{2}(k)=$ $d_{3}(k)=e^{-0.1 * k}$, the simulation results are plotted under the switching signal described in Figure 1. For the case of unavailable states, from Figures 3 and 4, we can see that the states $x(k)$ and the disturbance $d_{1}(k)$ can be estimated effectively by observers (10). With the gains in (72), Figure 2 shows that the system can be stabilized with a $H_{\infty}$ performance level by the composite controller (10). When the states are available, the response curves of System (1) and the disturbance errors are described in Figures 5 and 6, respectively. The stability of the closed-loop system under disturbance-observed-based controller (42) can be achieved with a $H_{\infty}$ performance level.

$$
P_{1}=\left[\begin{array}{ll}
1.5873 & 0.2634 \\
0.2634 & 1.5873
\end{array}\right]
$$




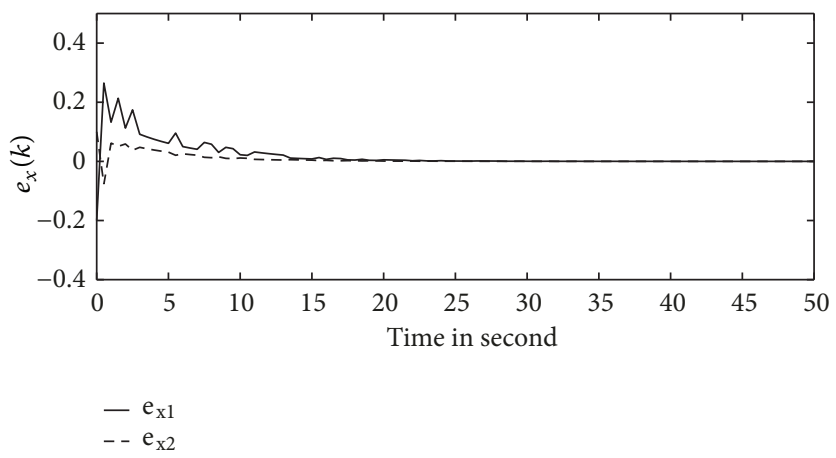

FIGURE 3: Curves of the state error $e_{d}(k)$ under full-order observer (10).

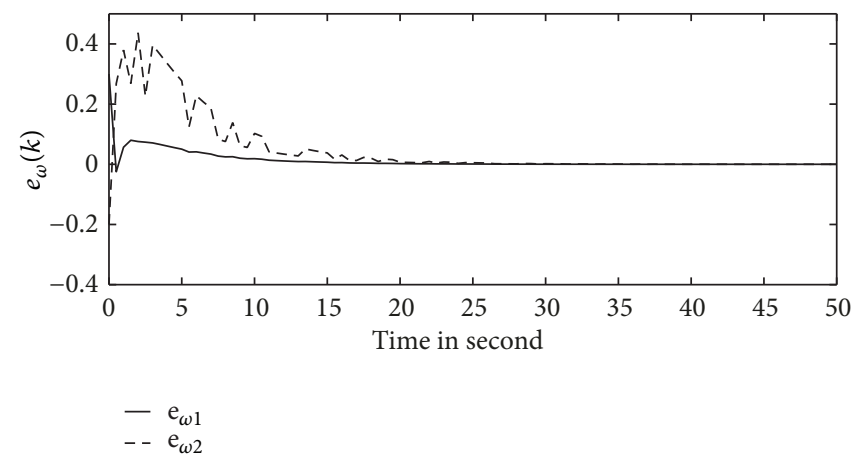

FIGURE 4: Curves of the disturbance error $e_{\omega}(k)$ under observer (10).

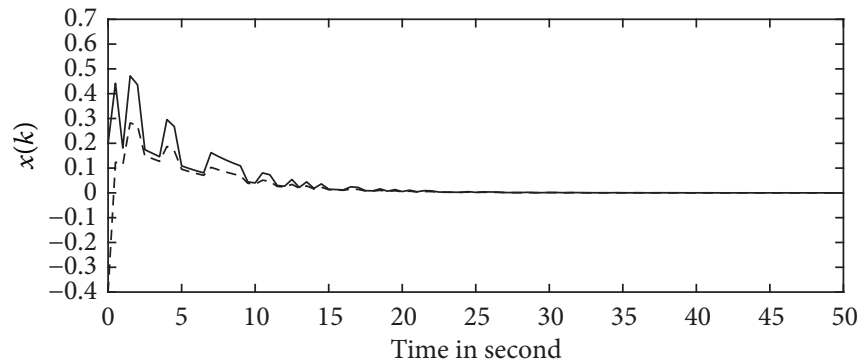

$$
\begin{aligned}
& -\mathrm{x}_{1} \\
& --\mathrm{x}_{2}
\end{aligned}
$$

FIGURE 5: The state trajectories of System (1) under disturbance-observer-based controller (42).

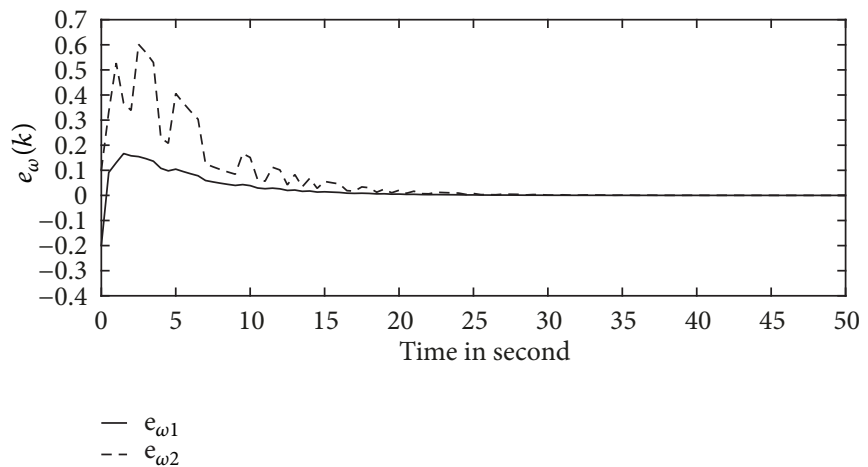

Figure 6: Curves of the disturbance error $e_{\omega}(k)$ under disturbance observer (42). 


\section{Conclusion}

The problem of analysis and synthesis of a class of switched nonlinear systems with saturation and multiple disturbances has been investigated in this paper. Based on designing the full-order and reduced-order observers, a composite controller is constructed to ensure the augmented system is robustly asymptotically stable in the absence of the disturbance. Further, a $H_{\infty}$ control scheme has been proposed to attenuate the mismatched disturbances.

\section{Data Availability}

The data used to support the findings of this study are available from the corresponding author upon request.

\section{Conflicts of Interest}

The authors declare that they have no conflicts of interest.

\section{Acknowledgments}

This work was supported in part by a research grant from the National Science Foundation of China: 61703233, Postdoctoral Science Foundation of China: 2016M602112, Natural Science Foundation of Shandong Province: ZR2016FQ09, the Open Fund of Key Laboratory of Measurement and Control of Complex Systems of Engineering, Ministry of Education under Grant MCCSE2016A04, Science and Technology Planning Project of Qufu Normal University: xkj201513, and National College Students' Innovation and Entrepreneurship Training Program: 201710446086.

\section{References}

[1] T.-C. Lee, Y. Tan, and I. Mareels, "Analyzing the stability of switched systems using common zeroing-output systems," Institute of Electrical and Electronics Engineers Transactions on Automatic Control, vol. 62, no. 10, pp. 5138-5153, 2017.

[2] M. T. Raza, A. Q. Khan, G. Mustafa, and M. Abid, "Design of fault detection and isolation filter for switched control systems under asynchronous switching," IEEE Transactions on Control Systems Technology, vol. 24, no. 1, pp. 13-23, 2015.

[3] H. Lin and P. J. Antsaklis, "Stability and stabilizability of switched linear systems: a survey of recent results," IEEE Transactions on Automatic Control, vol. 54, no. 2, pp. 308-322, 2009.

[4] L. Wu, R. Yang, P. Shi, and X. Su, "Stability analysis and stabilization of 2-D switched systems under arbitrary and restricted switchings," Automatica, vol. 59, article no. 6426, pp. 206-215, 2015.

[5] M. A. Bagherzadeh, J. Ghaisari, and J. Askari, "Robust exponential stability and stabilisation of parametric uncertain switched linear systems under arbitrary switching," IET Control Theory \& Applications, vol. 10, no. 4, pp. 381-390, 2016.

[6] X. Lin, C.-C. Chen, and C. Qian, "Smooth output feedback stabilization of a class of planar switched nonlinear systems under arbitrary switchings," Automatica, vol. 82, pp. 314-318, 2017.
[7] M. Philippe, R. Essick, G. E. Dullerud, and R. M. Jungers, "Stability of discrete-time switching systems with constrained switching sequences," Automatica, vol. 72, pp. 242-250, 2016.

[8] H. Yang, B. Jiang, G. Tao, and D. Zhou, "Robust stability of switched nonlinear systems with switching uncertainties," Institute of Electrical and Electronics Engineers Transactions on Automatic Control, vol. 61, no. 9, pp. 2531-2537, 2016.

[9] W. Xiang, H.-D. Tran, and T. T. Johnson, "Robust exponential stability and disturbance attenuation for discrete-time switched systems under arbitrary switching," IEEE Transactions on Automatic Control, vol. 63, no. 5, pp. 1450-1456, 2018.

[10] S. Dong, H. Su, P. Shi et al., "Filtering for discrete-time switched fuzzy systems with quantization," IEEE Transactions on Fuzzy Systems, vol. 25, no. 6, pp. 1616-1628, 2017.

[11] L. Li, L. Liu, and Y. Yin, "Stability analysis for discretetime switched nonlinear system under mdadt switching," IEEE Access, vol. 5, pp. 18646-18653, 2017.

[12] G. S. Deaecto, M. Souza, and J. C. Geromel, "Discrete-time switched linear systems state feedback design with application to networked control," Institute of Electrical and Electronics Engineers Transactions on Automatic Control, vol. 60, no. 3, pp. 877-881, 2015.

[13] F. Zhu, "Full-order and reduced-order observer-based synchronization for chaotic systems with unknown disturbances and parameters," Physics Letters A, vol. 372, no. 3, pp. 223-232, 2008.

[14] Y. Li, H. Sun, G. Zong et al., "Anti-disturbance control for timevarying delay Markovian jump nonlinear systems with multiple disturbances," International Journal of Systems Science, vol. 48, no. 7, pp. 1-15, 2017.

[15] S. Sun, X. Wei, H. Zhang, H. R. Karimi, and J. Han, "Composite fault-tolerant control with disturbance observer for stochastic systems with multiple disturbances," Journal of The Franklin Institute, vol. 355, no. 12, pp. 4897-4915, 2018.

[16] X. Yao and L. Guo, "Composite anti-disturbance control for Markovian jump nonlinear systems via disturbance observer," Automatica, vol. 49, no. 8, pp. 2538-2545, 2013.

[17] Y.-E. Wang, X.-M. Sun, and F. Mazenc, "Stability of switched nonlinear systems with delay and disturbance," Automatica, vol. 69, pp. 78-86, 2016.

[18] A.-T. Nguyen, T. Laurain, R. Palhares, J. Lauber, C. Sentouh, and J.-C. Popieul, "LMI-based control synthesis of constrained Takagi-Sugeno fuzzy systems subject to L2 or Lo disturbances," Neurocomputing, vol. 207, pp. 793-804, 2016.

[19] J. Hu, Z. Wang, F. E. Alsaadi, and T. Hayat, "Event-based filtering for time-varying nonlinear systems subject to multiple missing measurements with uncertain missing probabilities," Information Fusion, vol. 38, pp. 74-83, 2017.

[20] J. Yang, J. Sun, W. X. Zheng, and S. Li, "Periodic event-triggered robust output feedback control for nonlinear uncertain systems with time-varying disturbance," Automatica, vol. 94, pp. 324333, 2018.

[21] H. Shen, Y. Men, Z. Wu et al., "Nonfragile Ho control for fuzzy Markovian jump systems under fast sampling singular perturbation," IEEE Transactions on Systems, Man, and Cybernetics: Systems, vol. 99, pp. 1-12, 2017.

[22] P. Li, S. Xu, W. Chen, Y. Wei, and Z. Zhang, "A connectivity preserving rendezvous for unicycle agents with heterogenous input disturbances," Journal of The Franklin Institute, vol. 355, no. 10, pp. 4248-4267, 2018.

[23] H. Min, S. Xu, Y. Li, Y. Chu, Y. Wei, and Z. Zhang, "Adaptive finite-time control for stochastic nonlinear systems subject to 
unknown covariance noise," Journal of The Franklin Institute, vol. 355, no. 5, pp. 2645-2661, 2018.

[24] S. Tarbouriech, G. Garcia, J. M. G. da Silva Jr. et al., Stability and Stabilization of Linear Systems with Saturating Actuators, Springer Science \& Business Media, 2011.

[25] Y. Li and Z. Lin, "Saturation-based switching anti-windup design for linear systems with nested input saturation," Automatica, vol. 50, no. 11, pp. 2888-2896, 2014.

[26] Z. Wang, D. W. C. Ho, H. Dong et al., "Robust scrHsbinfty finite-horizon control for a class of stochastic nonlinear timevarying systems subject to sensor and actuator saturations," IEEE Transactions on Automatic Control, vol. 55, no. 7, pp. 17161722, 2010.

[27] M. Ran, Q. Wang, and C. Dong, "Stabilization of a class of nonlinear systems with actuator saturation via active disturbance rejection control," Automatica, vol. 63, pp. 302-310, 2016.

[28] J. Hu, Z. Wang, and H. Gao, "Joint state and fault estimation for time-varying nonlinear systems with randomly occurring faults and sensor saturations," Automatica, vol. 97, pp. 150-160, 2018.

[29] F. Wu and J. Lian, "A parametric multiple Lyapunov equations approach to switched systems with actuator saturation," Nonlinear Analysis: Hybrid Systems, vol. 29, pp. 121-132, 2018.

[30] A. Nguyen T, M. Dambrine, and J. Lauber, "Lyapunov-based robust control design for a class of switching non-linear systems subject to input saturation: application to engine control," IET Control Theory \& Applications, vol. 8, no. 17, pp. 1789-1802, 2015.

[31] J. Du, X. Hu, and Y. Sun, "Robust dynamic positioning of ships with disturbances under input saturation," Automatica, vol. 73, pp. 207-214, 2016.

[32] W. Qi, Y. Kao, X. Gao, and Y. Wei, "Controller design for time-delay system with stochastic disturbance and actuator saturation via a new criterion," Applied Mathematics and Computation, vol. 320, pp. 535-546, 2018.

[33] Y. Qian, Z. Xiang, and H. R. Karimi, "Disturbance tolerance and rejection of discrete switched systems with time-varying delay and saturating actuator," Nonlinear Analysis: Hybrid Systems, vol. 16, no. 1, pp. 81-92, 2015.

[34] Y. Qin, Y. Liang, Y. Yang, Q. Pan, and F. Yang, "Minimum upperbound filter of Markovian jump linear systems with generalized unknown disturbances," Automatica, vol. 73, pp. 56-63, 2016.

[35] Z. Yu, H. Yan, S. Li, and Y. Dong, "Adaptive quantised control of switched stochastic strict-feedback non-linear systems with asymmetric input saturation," IET Control Theory \& Applications, vol. 12, no. 10, pp. 1367-1375, 2018.

[36] Y. Wei, W. X. Zheng, and S. Xu, "Anti-disturbance control for nonlinear systems subject to input saturation via disturbance observer," Systems \& Control Letters, vol. 85, pp. 61-69, 2015. 


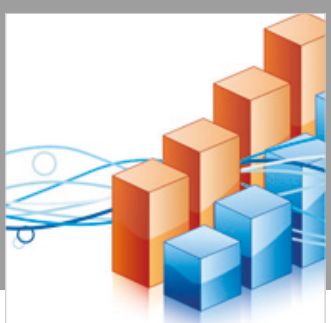

Advances in

Operations Research

\section{-n-m}
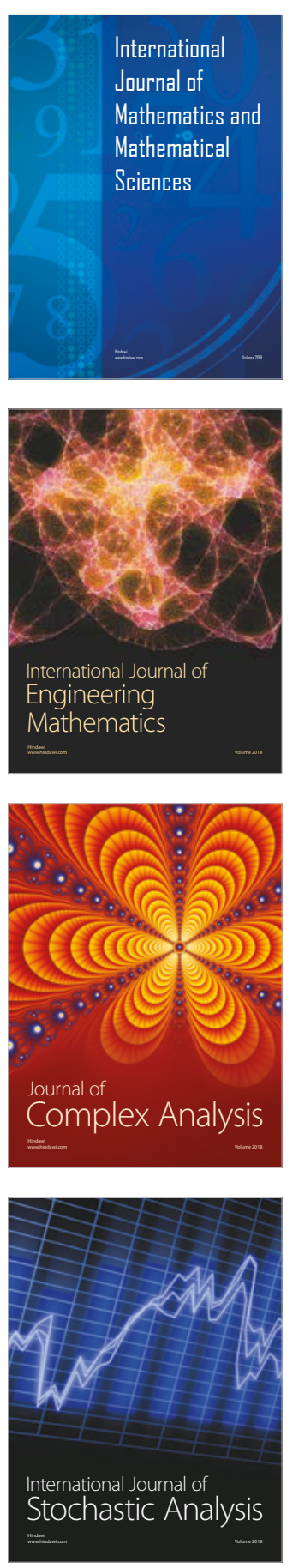
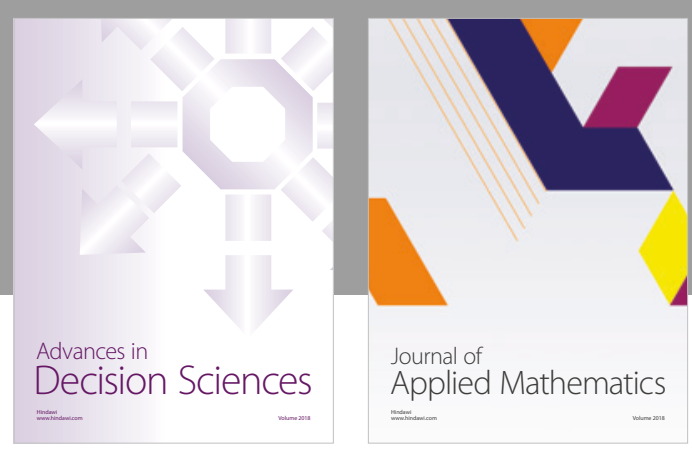

Journal of

Applied Mathematics
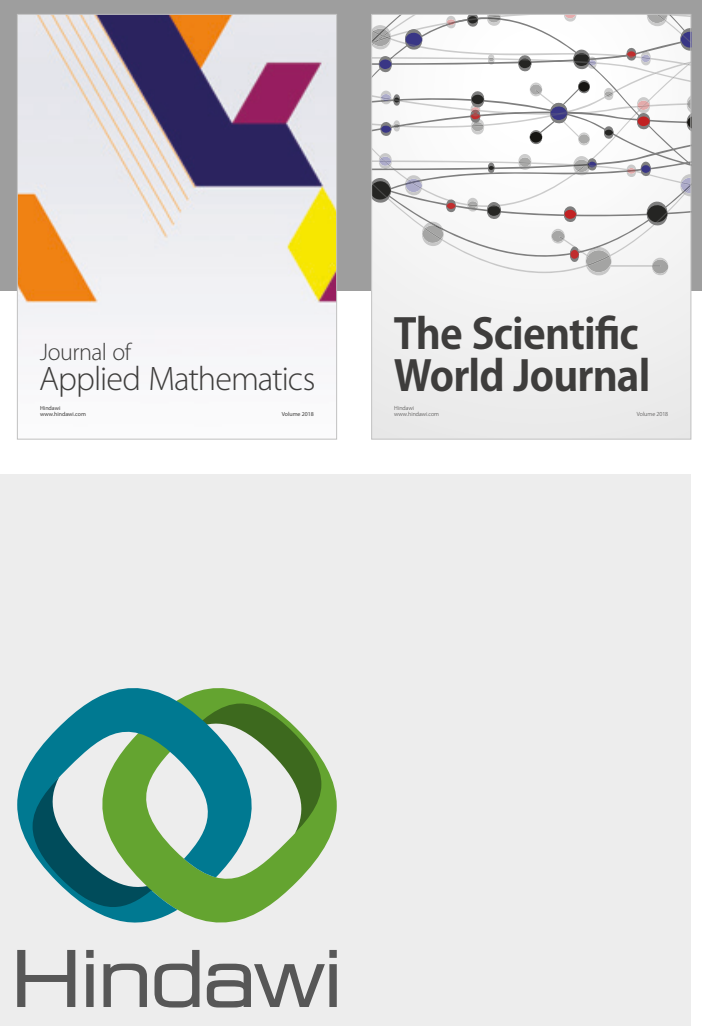

Submit your manuscripts at

www.hindawi.com

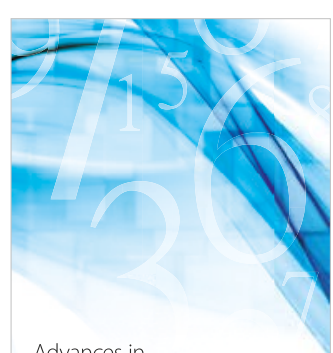

Advances in
Numerical Analysis
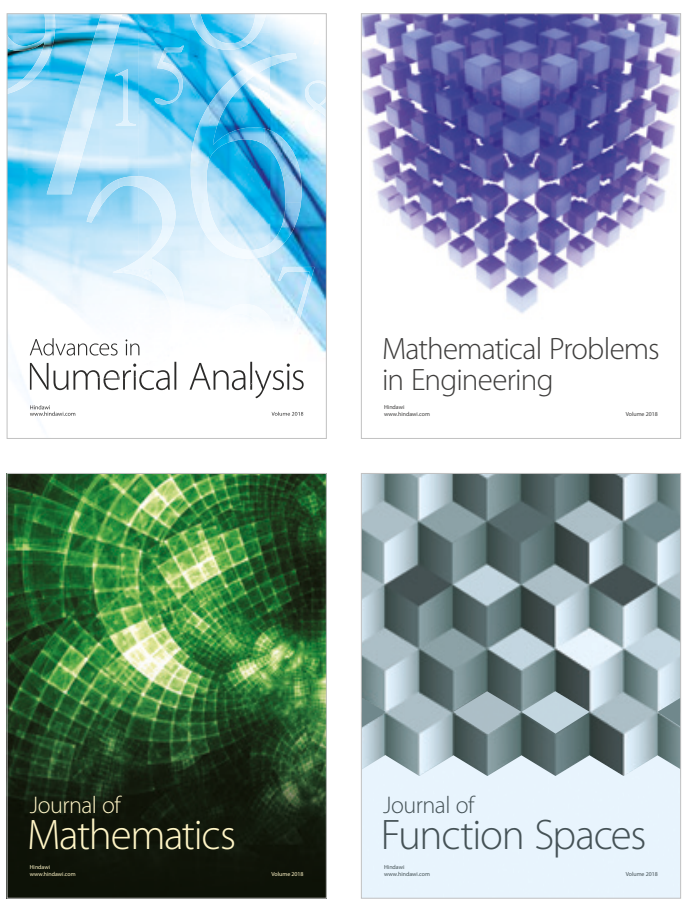

Mathematical Problems in Engineering

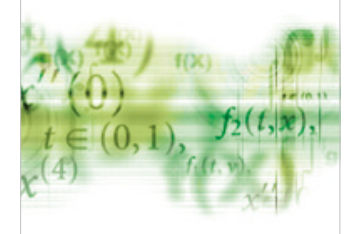

International Journal of

Differential Equations

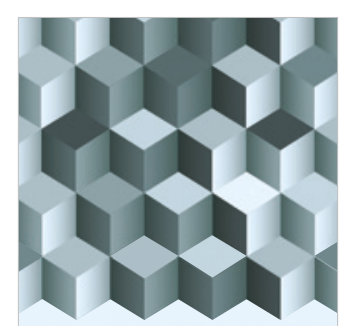

Journal of

Function Spaces

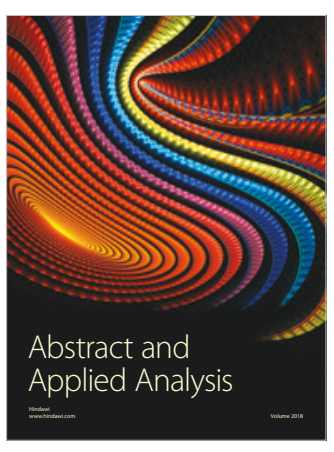

The Scientific

World Journal

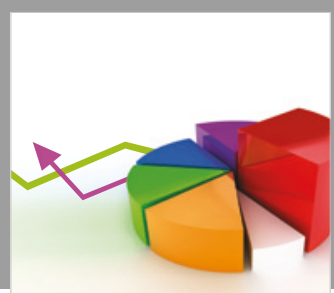

Journal of

Probability and Statistics
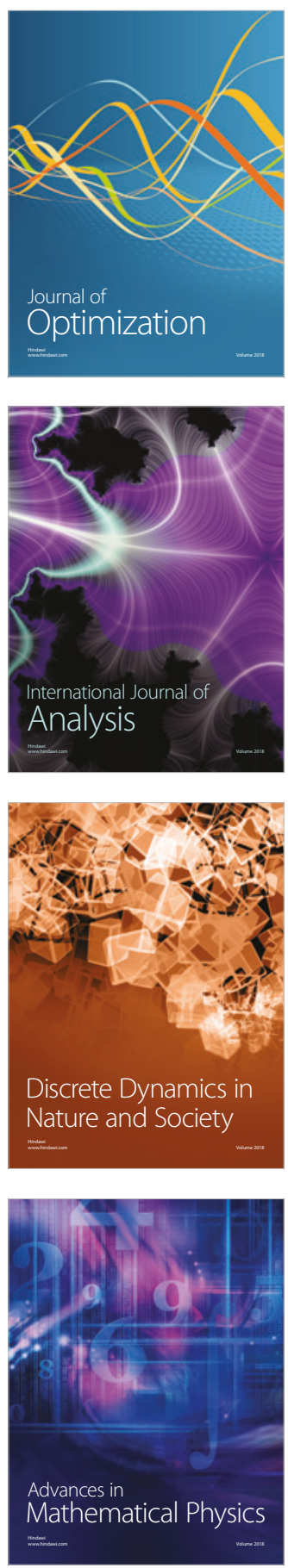